\title{
MODELLING OF HOLE SINKING ELECTRICAL DISCHARGE MICRO MACHINING OF NI-BASED SUPER ALLOY THIN SHEET
}

\begin{tabular}{|c|c|c|}
\hline $\begin{array}{c}\text { Rajesh Kumar Porwal } \\
\text { Department of Mechanical Engineering } \\
\text { KCC Institute of Technology \& Mgmt. } \\
\text { Gr. Noida, India }\end{array}$ & $\begin{array}{c}\text { Sanjay Mishra } \\
\text { Department of Mechanical Engineering } \\
\text { I.T.S Engineering College } \\
\text { Gr. Noida, India }\end{array}$ & $\begin{array}{c}\text { Basanta Kumar Bhuyan } \\
\text { Department of Mechanical Engineering } \\
\text { Manav Rachna International University } \\
\text { Faridabad, India }\end{array}$ \\
\hline
\end{tabular}

\begin{abstract}
From the productivity and accuracy point of view fabrication of the symmetrical and nonsymmetrical holes is a challenging task for manufacturing engineers. Creation of these kinds of holes in thin sheets of super alloys can only be possible by using hole sinking electro discharge micro machining (HSEDMM) process. In the present paper Artificial Neural Network (ANN) model has been proposed for the prediction of material removal rate (MRR) in HS-EDMM. For this purpose Matlab with the neural networks toolbox (nntool) has been used. The neural network based on process model has also been developed to establish relationship between input process conditions (gap voltage and capacitance of the capacitor) and process response material removal rate. The ANN model has been trained and tested using the data generated from an extensive series of experiments on HS-EDMM machine. The trained neural network system has been used to predict MRR for different input conditions. The ANN model has been found to predict accurately HS-EDMM process responses for chosen process conditions.
\end{abstract}

Keywords - Hole sinking electrical discharge micro machining (HSEDMM), Artificial neural network (ANN), Back-propagation (BP) algorithm.

\section{INTRODUCTION}

In recent years, there is an increasing trend towards miniaturization of various engineering product/or product components. Micromachining is the one of the machining methods for the production of miniaturized parts and components based on different mechanism of material removal. These miniaturized components/ products having multi functional characteristics are largely employed in electronics, optics, automobile, biotechnology, and aeronautical industries. Electrical discharge micromachining (EDMM) is one of the successful micromachining processes having capability to create micro features from simple to complex shapes in electrically conductive workpiece materials. It has been known that the material removal mechanism in EDMM is due to plasma formation, heat transfer, superheating of electrodes and ejection of material due to electrical discharge energy transfer of the order of less than hundreds of micro joules. The power supply used in EDMM is either relaxation type pulse generator or transistor type pulse generator with $\mathrm{MHz}$ pulsating frequency. Various gap monitoring and control strategies have been developed to avoid any problem of arcing and short circuiting.

In view of this, micromachining techniques have become important in the fabrication of micro components based on the different mechanism of material removal. Based on the different forms of innovative energy utilizing in micromachining processes are classified into various categories like electro discharge micromachining (EDMM) using electrical discharge spark energy, beam micromachining processes (BMMPs) using beam of photon, electron or ions, electrochemical micromachining (ECMM) using controlled electrolysis, chemical micromachining processes (CMMPs) using selective chemical reaction, ultrasonic micromachining (USMM) using high frequency vibrational energy and jet micromachining processes (JMMPs) using energy of jet.

\section{LITERATURE REVIEW}

The origin of EDM can be traced as far back as 1770, when English chemist Joseph Priestly discovered the erosive effect of electrical discharge. However it was only in 1943 at the Moscow University where Lazarenko and Lazarenko exploited the destructive properties of electrical discharges for constructive use. They developed a controlled process of machining difficult to machine metals by vaporizing material from the surface of metal. Wong et al. have developed a singlespark generator to study the erosion characteristics from the micro crater size due to EDMM. Their experimental results suggested that volume and size of the micro craters are found to be more consistent at lower-energy discharges than at higherenergy discharges [1]. An optical sensor has been developed by Lim et al. to measure and control the dimension of the thin electrode during the tool fabrication process. They observe that the rotating electrode shows the best performance in the highaspect ratio tool-electrode fabrication and machining depth is inversely proportional to the feed rate [2]. A 3-axis local actuator module for EDMM was developed by Yoshihito et al. This module had $200 \mathrm{~Hz}$ bandwidth and utilized the electromagnetic force for the holding and positioning of the electrode. A $60 \mu \mathrm{m}$ diameter micro-hole with aspect ratio over 16 was machined by this module [3]. Fuzhu et al. has developed a new transistor type isopulse generator and servo feed control to improve the machining characteristics of EDMM. It is observed that the transistor type isopulse generator is more useful in semi finishing than in finishing, whereas servo feed control is better in finishing as compared to semi finishing [4].

The factors which may affect minimum smallest possible rod diameter that can be obtained by EDMM was investigated by Fuzhu et al. It was observed that tungsten carbide and cemented tungsten carbide are better than tungsten because of 
both crystal grain size and size of defects among grains are smaller. The surface finish obtained with reverse polarity is better than straight polarity [5]. Jahan et al. studied the effect of different tool electrode materials (W, $\mathrm{AgW}, \mathrm{CuW}$ ) on workpiece material (WC) for material removal rate (MRR) and tool wear rate (TWR). It was observed that the $\mathrm{AgW}$ electrode produces smoother and defect-free nano surface among the three electrodes. Besides, a minimum amount of material migrates from the $\mathrm{AgW}$ electrode to the WC workpiece during the finishing EDMM. CuW electrode achieved highest MRR while $\mathrm{W}$ electrode has lowest tool wear among all electrodes [6]. A new technique to measure the volume of material removed from the tool and the workpiece was proposed by Karthikeyan et al. It has been found that the rotation speed plays a significant role in achieving the desired MRR by enhancing flushing. The surface analysis shows that the debris formed by low value of speed and feed rate have resulted in bigger size debris at the edges [7].

Indurkhya and Rajurkar have developed a 9-9-2-size back-propagation neural network for orbital EDM modeling. Having compared the results of the neural network model with estimates obtained via multiple regression analysis, they concluded that the neural model is more accurate and also less sensitive to noise included in the experimental data [8]. Assarzadeh and Ghoreishi have presented a new integrated neural network based approach for the prediction and optimal selection of process parameters in die sinking electro-discharge machining with a flat electrode [9]. Basheer et al. have investigated the roughness of machined surfaces on $\mathrm{Al} / \mathrm{SiC}$ metal metrix composites and developed an ANN-based model to predict surface roughness of machined surfaces using a feedforward network and an algorithm involving Bayesian regularization combined with the Levenberg-Marquardt modification to train the neural network [10].

The present paper attempts to develop a feed forward BPNN model for the prediction of material removal rate (MRR) in HS-EDMM machined holes. The developed model is used for the study of the effect of process parameters of HSEDMM process such as gap voltage and capacitance of capacitor on performance parameter such as material removal rate.

\section{EXPERIMENTAL PLANNING}

Hole sinking electrical discharge micromachining (HSEDMM) was performed on multi process micro electro discharge machine (Model DT-100, Mikrotool Pte, Singapore), having fixed level of capacitance of capacitor, and adjustable range of voltage. Tungsten carbide rod of 500micron diameter was used as tool electrode. The micro HS-EDMM operation was performed on rectangular section cuboid shape workpiece specimens made of Invar-36 having mean thickness of $0.5 \mathrm{~mm}$, length $25 \mathrm{~mm}$, and width $15 \mathrm{~mm}$. The removal of debris was achieved by lateral flushing with dielectric (EDM oil). The depth of cut was kept constant 510 micron for all experiments. In the present research, analysis of the effect of different parameter settings on material removal rate was carried out through artificial neural network (ANN) modelling. After preliminary investigations, two process parameters were selected as gap voltage, capacitance of capacitor because they directly affect the performance parameters such as material removal rate. Selection of the range of process parameter settings was made after performing some pilot experiments within the stable domain of the machining. The levels of parameters selected are shown in Table (1). The amount of material removed from the workpiece was measured with the help of citizen make micro weighing balance

Table 1 the machining parameters considered and their levels

\begin{tabular}{llllllll} 
Machining & Unit & \multicolumn{7}{c}{ Levels } \\
parameters & & 1 & 2 & 3 & 4 & 5 & 6 \\
\cline { 3 - 7 } Voltage & $\mathrm{V}$ & 90 & 100 & 110 & 120 & 130 & 140 \\
Capacitance & $\mathrm{nF}$ & 10 & 100 & 400 & & &
\end{tabular}

having least count of 0.0001 grams. Material removal rate (MRR) is defined as volume of material removed in unit time from workpiece. Hence, based on their density the MRR is calculated as;

$\operatorname{MRR}=\frac{\text { Massof workpiece material removed }}{\text { Density of workpiece material } \times \text { Time tomake hole }}$

The values of MRR are calculated by using equation (1) based on experimental results. The process parameters, the corresponding MRR are again normalized using equation (2). $X=2 \times \frac{\left(R-R_{\min }\right)}{\left(R_{\max }-R_{\min }\right)}-1$,

where $X$ is the normalized value of real variable, $R$ is the real value of the variable, and $R_{\max }$ and $R_{\min }$ are the maximum and minimum values of the real variable. Normalization of the variables is in the range of -1 to +1 by using equation (2). These normalized data set as given in table (2) were used for training the network. The microscopic view of micro drilled hole is shown in Fig.1.

Experiments were carried out using fractional factorial combinations of these factors and their different levels. During experiments the workpiece thickness was kept constant for all experimental run. Dielectric was also kept same for experimentation. As per Taguchi methodology an orthogonal array was selected based on the input parameters and their levels. Interaction effect was not taken into account. $\mathrm{L}_{18}$ orthogonal array was selected with capacitance of capacitor as three levels and gap voltage of six levels. To achieve validity and accuracy, each test was repeated three times and averages of it were considered in this study 


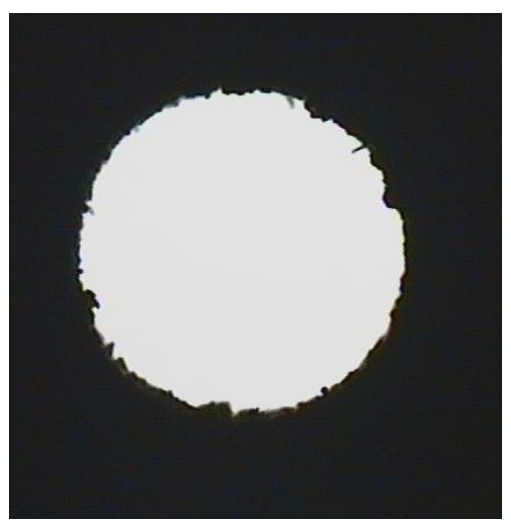

Fig. 1 Microscopic views of micro drilled hole (120 V and 400 $\mathrm{nF} 10 \mathrm{x}$ magnifications).

The responses considered were material removal rate of the micro drilled through holes. Based on the experimental inputoutput results an ANN model was developed through training and testing of the data set for input-output mapping. All the responses were set as targets while training of the network was ongoing, Table (2).

Table 2 Single dataset for the neural network model (The values of variables are normalized)

\begin{tabular}{cccc}
\hline $\begin{array}{l}\text { Exp. } \\
\text { No. }\end{array}$ & Voltage & Capacitance & $\begin{array}{l}\text { Material removal } \\
\text { rate }\end{array}$ \\
\hline 1 & -1.0000 & -1.0000 & -0.9964 \\
2 & -1.0000 & -0.5385 & -0.7148 \\
3 & -1.0000 & 1.0000 & 0.0444 \\
4 & -0.6000 & -1.0000 & -0.9771 \\
5 & -0.6000 & -0.5385 & -0.6401 \\
6 & -0.6000 & 1.0000 & 0.4239 \\
7 & -0.2000 & -1.0000 & -0.9332 \\
8 & -0.2000 & -0.5385 & -0.6268 \\
9 & -0.2000 & 1.0000 & 0.7535 \\
10 & 0.2000 & -1.0000 & -0.9333 \\
11 & 0.2000 & -0.5385 & -0.3028 \\
12 & 0.2000 & 1.0000 & 0.8697 \\
13 & 0.6000 & -1.0000 & -0.8922 \\
14 & 0.6000 & -0.5385 & -0.2895 \\
15 & 0.6000 & 1.0000 & 0.9272 \\
16 & 1.0000 & -1.0000 & -0.8790 \\
17 & 1.0000 & -0.5385 & -0.2133 \\
18 & 1.0000 & 1.0000 & 1.0000 \\
\hline
\end{tabular}

The Levenberg-Marquadt (LM) algorithm was used as training algorithm, as it is the fastest and consumes the least memory [11]. The neural network toolbox of MATLAB software was used for modelling. After proper training of the network, the network was simulated with other input parameter combinations and the network responses compared with the experimental responses, Table (3). Thus validation of the developed model has been checked. The optimum parameter setting for the desired responses is then found by using the responses of the network model.

\section{ANN MODELLING OF HS-EDMM PROCESS}

Modelling of machining, aimed at better understanding of process, has been a subject of interest for researchers in the past years. As a result, modelling of machining processes has been examined from different points of view by using different techniques. Large number of parameters, partially understood relations between parameters, complex, multi-dimensional, non-linear and

Table 3 Comparison of the developed model with experimental data and the errors in prediction

\begin{tabular}{cccccc}
\hline $\begin{array}{c}\text { Exp } \\
\text { No. }\end{array}$ & Volt. & Cap. & $\begin{array}{c}\text { Exp. } \\
\text { MRR x } 10^{-4} \\
\left(\mathrm{~mm}^{3} / \mathrm{min}\right)\end{array}$ & $\begin{array}{l}\text { ANN } \\
\text { predicted } \\
\text { MRR }\end{array}$ & $\begin{array}{c}\text { \% error in } \\
\text { prediction of } \\
\text { MRR } \\
\text { (absolute) }\end{array}$ \\
\hline 1 & 90 & 10 & 2.5385 & 2.5894 & 2.01 \\
2 & 90 & 100 & 12.15 & 11.7972 & 2.90 \\
3 & 90 & 400 & 44.8671 & 46.1449 & 2.85 \\
4 & 100 & 10 & 3.2116 & 2.9877 & 6.97 \\
5 & 100 & 100 & 14.7595 & 14.4408 & 2.16 \\
6 & 100 & 400 & 51.4352 & 49.2544 & 4.24 \\
7 & 110 & 10 & 4.7865 & 4.822 & 0.74 \\
8 & 110 & 100 & 15.3243 & 16.1997 & 5.71 \\
9 & 110 & 400 & 63.2314 & 62.0897 & 1.81 \\
10 & 120 & 10 & 4.8657 & 5.1122 & 5.07 \\
11 & 120 & 100 & 26.7812 & 24.918 & 6.96 \\
12 & 120 & 400 & 66.9458 & 69.7826 & 4.24 \\
13 & 130 & 10 & 6.1796 & 6.0979 & 1.32 \\
14 & 130 & 100 & 27.5459 & 29.1167 & 5.70 \\
15 & 130 & 400 & 68.7956 & 68.0922 & 1.02 \\
16 & 140 & 10 & 16.5897 & 17.0781 & 2.94 \\
17 & 140 & 100 & 28.8996 & 30.1818 & 4.44 \\
18 & 140 & 400 & 71.9547 & 72.0446 & 0.12 \\
\hline & Total average prediction error $(\%)=3.4$ & \\
\hline & & & & & \\
\hline
\end{tabular}

stochastic nature of machining offer modeling of machining processes a considerable task. In this respect Neural network proves itself as a suitable modelling tool by possessing many characteristics which make it suitable for addressing such responsibilities like universal function approximation capability, resistance to noisy or missing data, accommodation of multiple nonlinear variables for unknown interactions, and good generalization capability and adaptive nature. These capabilities of neural network are of primarily significance for modelling of HS-EDMM process. The superiority of using neural networks in modelling of machining processes has been reported in several studies (Tarng et al. [12], Dilma et al. [13], and Porwal et al.[14, 15]). The block diagram of modelling of ANN for the present work is shown in fig. 2. A neural network can be viewed as a function that maps input vectors to output vectors. The knowledge is presented by the interconnection weight, which is adjusted during the learning stage. In present study Matlab ${ }^{\circledR}$ with the neural networks 


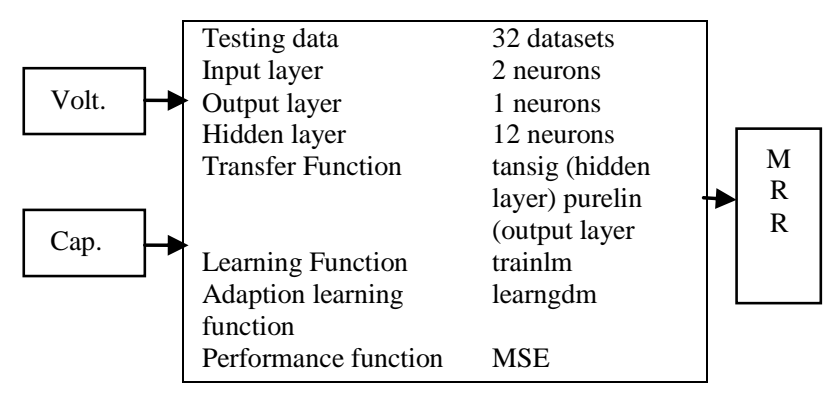

Fig. 2 Neural network specifications

toolbox (nntool) is used for formulation of artificial neural network and modeling of HS-EDMM. Matlab ${ }^{\circledR}$ is a well-known program used for modeling purposes. Its neural network toolbox is user-friendly and the creation of neural networks is performed by using a small amount of commands [11]. The program has a data base with functions, algorithms and commands for this purpose. In general, a neural network is characterized by its important features such as the architecture, the activation function and the learning algorithms. Several models were designed and tested with process parameter in order to determine the optimal architecture, the most suitable activation function and the best training algorithm suitable for the prediction of MRR. Each model was tested more than once in order to assess whether it truly congregates at which point when the prediction error and mean square error were a minimum, Fig.3. The value of prediction error is the absolute value of ratio of difference of experimental value and predicted value to the experimental value.

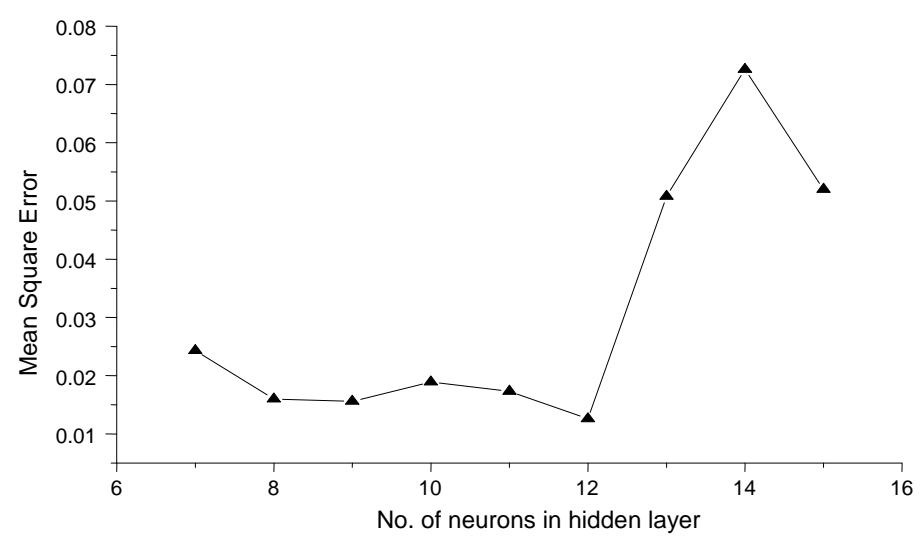

Fig. 3 Effect of number of neurons in hidden layer on mean square error

After exhaustive number of trial and error procedure the model selected was a feed forward neural network with single hidden layer consisting of 12 neurons. Therefore a network of structure 2-12-1 is found to be the most suitable network for the present task. The activation function in the hidden layer was the hyperbolic tangent sigmoid transfer function (tansig) and in the output layer was the linear transfer function (pureline). The learning algorithm used was the back propagation algorithm.
Back propagation is a systematic method for training multilayer artificial neural network [14]. It uses gradient-descent method to minimize the total mean square error of the output computed by the network. The developed model is shown schematically in Fig. 4. The values of MRR is calculated by using equation (1) and subsequently normalized by using equation (2). Two third of the normalized experimental results are used to train the neural network, and rest is for testing. Selection of data for training and testing is in random fashion. Properly trained backpropagation network tends to give reasonable answers when presented with inputs that have never been fed before to it.

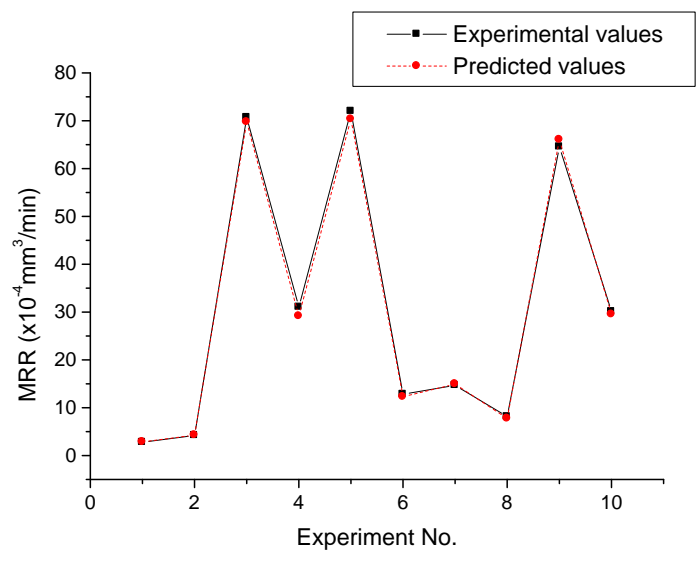

Fig. 4 Comparison of the material removal rate between validation and experimental result

An experimental approach was adopted that involved testing the trained neural network against another set of experimental data, illustrated in Table (3). The errors in prediction are also presented in Table (3). It can be seen from Table (3) that the model predicted data followed the experimental data very closely except few calculations. It can be said that the error is within the tolerable limit. Moreover, the average error in the prediction was $3.4 \%$ for MRR, which were very small indeed.

Table 4 Verification of the developed model with experimental data

\begin{tabular}{cccccc}
\hline $\begin{array}{c}\text { Exp } \\
\text { No. }\end{array}$ & Volt. & Cap. & $\begin{array}{c}\text { Exp. } \\
\text { MRR x 10 } \\
\left(\mathrm{mm}^{3} / \mathrm{min}\right.\end{array}$ & $\begin{array}{c}\text { ANN } \\
\text { predicted } \\
\text { MRR }\end{array}$ & $\begin{array}{c}\text { \% error in } \\
\text { prediction of } \\
\text { MRR } \\
\text { (absolute })\end{array}$ \\
\hline 1 & 96 & 10 & 2.7876 & 2.8515 & 2.29 \\
2 & 106 & 10 & 4.1963 & 4.2756 & 1.89 \\
3 & 116 & 400 & 70.6845 & 69.7659 & 1.30 \\
4 & 124 & 100 & 31.0436 & 29.1670 & 6.04 \\
5 & 136 & 400 & 71.9857 & 70.3459 & 2.28 \\
6 & 94 & 100 & 12.8176 & 12.2985 & 4.05 \\
7 & 104 & 100 & 14.6941 & 14.9512 & 1.75 \\
8 & 115 & 10 & 8.1352 & 7.7608 & 4.60 \\
9 & 126 & 400 & 64.549 & 66.0272 & 2.29 \\
10 & 134 & 100 & 30.0951 & 29.5262 & 1.89 \\
\hline
\end{tabular}


The developed ANN model was validated with the new set of experimental data (unseen) for same output features. Table (4) contains the predicted output and percentage error in prediction of MRR. It is observed that the total average prediction error is $3.03 \%$ which is within the tolerable limit. Generalization capabilities of the selected network can be easily verified by comparing the experimental results with modelling results; Fig. 4.

\section{PARAMETRIC STUDY}

Developed ANN model (2-12-1) is used for the study of the effect of input parameters of HS-EDMM process such as gap voltage and capacitance on performance parameter material removal rate. In the present study the effect of gap voltage and capacitance of capacitor on performance parameter has been discussed as follows.

\section{A. Effect of Gap Voltage on MRR}

Fig. 5 shows the variation of MRR with the gap voltage. It is observed that MRR increases with the increase in the gap voltage. The increase in gap voltage refers the rise of discharge energy in the machining zone. It is also observed that more material will get melted in unit time on the higher value of discharge energy. 50.4\% increase in MRR is observed when gap voltage changes from $90 \mathrm{~V}$ to $140 \mathrm{~V}$.

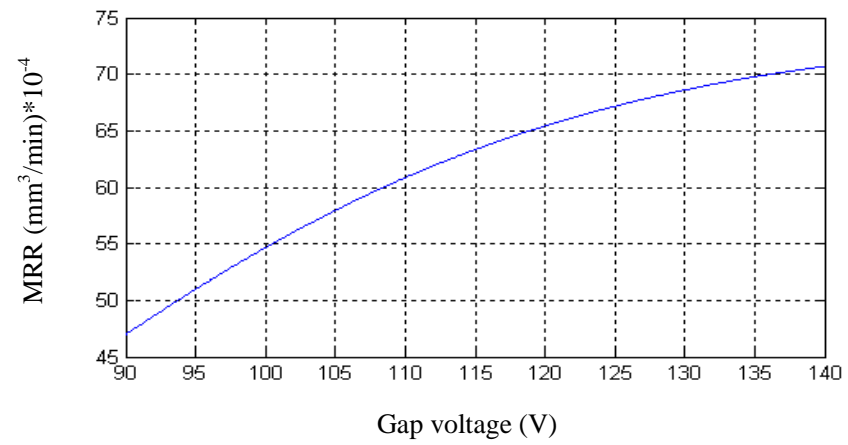

Fig. 5 Variation of MRR with the gap voltage

B. Effect of Capacitance on MRR

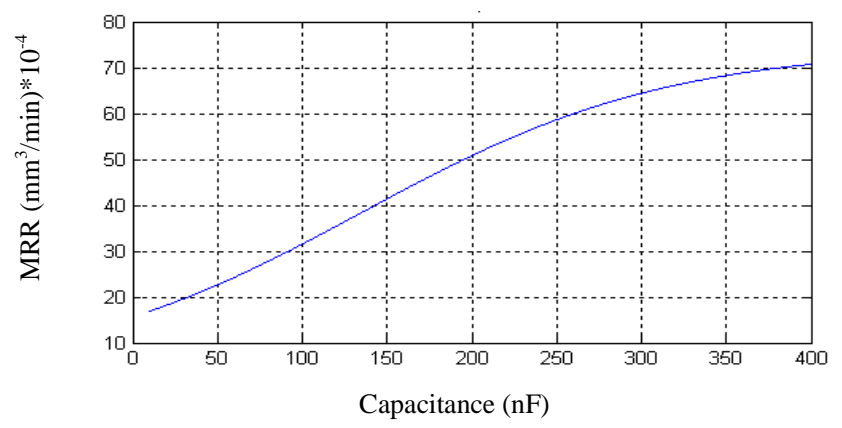

Fig. 6 Variation of MRR with the capacitance
The variation of MRR with the capacitance of capacitor is shown in fig. 6. This figure shows that the MRR increases with increase of capacitance. There is 319\% increase in MRR which is observed when capacitance of capacitor changes from $10 \mathrm{nF}$ to $400 \mathrm{nF}$. The increase in capacitance refers the raise of discharge energy in the machining zone which results more material getting melted and ejected in unit time. It is also observed that after $259 \mathrm{nF}$ capacitance the rate of increase in MRR is small as compared to before of it.

\section{CONCLUSIONS}

The results of present study are summarized as follows:

It has been found that among several neural configurations a feed-forward back-propagation ANN of type 2-12-1 having single hidden layer with ten neurons can provide the best prediction.

* The average error in the prediction of developed model was very small indeed. It was $3.40 \%$ for MRR, also the total average prediction error was $3.03 \%$.

* It is observed that the capacitance of capacitor has stronger effect on MRR as compared to gap voltage.

* It is found that the value of MRR is increased by $50.4 \%$ when voltage changes from $90 \mathrm{~V}$ to $140 \mathrm{~V}$.

* There is $319 \%$ increase in MRR which is observed when capacitance of capacitor changes from $10 \mathrm{nF}$ to $400 \mathrm{nF}$.

\section{References}

[1] Wong, Y.S., Rahman M., Lim, H.S., Han, H. and Ravi, N. "Investigation of micro-EDM material removal characteristics using single RC-pulse discharges", J. of Material Processing Technology, Vol. 140, pp. 303-307, 2003.

[2] Lim H.S., Y.S. Wong, M. Rahman and M.K. Edwin Lee, “A study on the machining of high-aspect ratio micro-structures using micro-EDM, J. of Materials Processing Technology", Vol. 140, pp. 318-325, 2003

[3] Yoshihito I., Takayuki N., Hidetaka M., Hirofumi H. and Hitoshi T., "Local actuator module for highly accurate micro-EDM", J. of Materials Processing Technology, Vol. 149, pp. 328-333, 2004.

[4] Fuzhu H., Shinya W. and Masanori K., "Improvement of machining characteristics of micro-EDM using transistor type isopulse generator and servo feed control", J. of Precision Engineering, Vol. 28, pp. 378-385, 2004

[5] Fuzhu H., Yuji Y., Taichi K., and Masanori K., "Experimental attempts of sub-micrometer order size machining using micro-EDM", J. of Precision Engineering, Vol. 30, pp.123-131, 2006

[6] Jahan M.P., Wong Y.S. and Rahman M. "A study on the fine-finish diesinking micro-EDM of tungsten carbide using different electrode materials", J. of Materials Processing Technology, Vol. 209, pp. 39563967, 2009

[7] Karthikeyan G., Ramkumar J., Dhamodaran S., Aravindan S., "Micro electric discharge milling process performance: An experimental investigation", Int. Journal of Machine Tools \& Manufacture, Vol. 50, pp. 718-727, 2010

[8] Indurkhya G. and Rajurkar K.P., "Artificial neural network approach in modeling of EDM process", Proceedings of the Artificial Neural Networks in Engineering, Conference, St. Louis, Missouri, 845-850, November 1992

[9] Assarzadeh S. and Ghoreishi M. "Neural-network-based modeling and optimization of the electro-discharge machining process", Int. Journal of Advanced Manufacturing Technology, Vol. 39, pp. 488-500, 2008

[10] Basheer A. C., Uday A. D., Joshi S. S., Bhanuprasad V.V., and Gadre V.M. "Modeling of surface roughness in precision machining of metal matrix composites using ANN", J. of Materials Processing Technology, Vol.197, pp. 439-444, 2008 
[11] Fausett, L.V., "Fundamentals of neural networks: architectures, algorithms and applications", Prentice-Hall ,New York, 1994

[12] Tarng, Y.S., Ma S.C. and Chung L.K., "Determination of optimal cutting parameters in wire electric discharge machining" Int. Journal of Machine Tools and Manufacture, Vol. 35, pp. 1693-1701, 1995

[13] Dilma Jr. D.E., Lsiter, P.M. and Leighton, N.J., "Neural network solutions to the tool condition monitoring problem in metal cuttingcritical review of methods" Int. Journal of Machine Tools and Manufacture, Vol. 37, pp. 1219-1241, 1997

[14] R. K. Porwal, V. Yadava and J. Ramkumar, "Artificial neural network modelling and multi objective optimization of hole drilling electro discharge micro machining of invar", Int. Journal of Mechatronics and Manufacturing Systems, Vol. 5, pp. 264-289, 2012

[15] R. K. Porwal, V. Yadava and J. Ramkumar, "Modelling and Optimization of Hole Drilling Electrical Discharge Micromachining Process of Ti-6Al4V Thin Sheet", Int. Journal of Precision Technology, Vol. 3, pp. 183205,2013 